\title{
Control and Reconfiguration of Train of Autonomous Electric Vehicles
}

\author{
Kamel Bouibed ${ }^{1}$, Abdel Aitouche ${ }^{2}$, and Mireille Bayart ${ }^{3}$ \\ ${ }^{1}$ CReSTIC, Université de Reims Champagne-Ardenne, kamel.bouibed@univ-reims.fr \\ ${ }^{2}$ LAGIS, Hautes Etudes d'Ingénieur (HEI Lille), abdel.aitouche@hei.f \\ ${ }^{3}$ LAGIS, Université Lille1 Sciences et Technologies, mireille.bayart@univ-lille1.fr
}

\begin{abstract}
This paper presents the concept of a convoy of autonomous electric vehicles which will be able to continue its mission in the presence of some actuator or sensor faults. The autonomous vehicle is called RobuCar which is constituted by four independently driven wheels and two independently adjustable steering angles. Firstly, we develop longitudinal and lateral controllers for the vehicles operating normally. The objective is to follow a reference trajectory and to maintain safe spacing between vehicles. Secondly, we develop some strategies based on fault detection and isolation results in order to reconfigure the convoy of vehicles in presence of faults. Three categories have been considered: sensor or actuator fault which has not altered the trajectory; fault that affects the velocity or disturbs the trajectory; faults that make impossible for the vehicle to continue its mission. Simulation results are given at the end to show the efficiency of the approach.
\end{abstract}

\section{Keywords}

autonomous electric vehicles, fault tolerant control, longitudinal and lateral control, trajectory following, reconfiguration, sliding mode control

\section{INTRODUCTION}

It has become necessary to develop new intelligent and clean transportation systems [Broggi et al., 1999; Taleb et al., 2010] to solve the problems of congestion in cities and facilitate mobility of citizens and reduce pollution caused by vehicles. Various projects have been devoted to the development of individual or collective transport systems using electric vehicles for several years. CRISTAL project [Colin et al., 2008, 2009] in France, concerns the development of new type of urban vehicles with new functionalities and services. One of its major cornerstones is the development, validation and certification of platoon of vehicles. A platoon is a set of autonomous vehicles which have to move in a convoy, i.e. follow the path of the leader through an intangible hooking. The project Praxitèle [Massot et al., 1999] concerns a transportation system consisting of a fleet of electric cars for public use, dispersed over a limited area and made available for free service. These cars are equipped with the electronic management, control and communication. The University of California-Riverside and Honda Motor Company have implemented in the USA a shared electric vehicle system test bed named UCR IntelliShare [Barth and Todd, 2000]. It consists of 15 shared electric vehicles moving among three stations near the UC Riverside campus. The system uses high- ly developed intelligent transportation system technology, including smart cards, touch-screen registration kiosks, vehicle monitoring and tracking hardware, and sophisticated management software. The system has operated since April 1999. These works are essentially focused on instrumentation, location, power, and control of these vehicles alone or in a convoy [Swaroop et al., 2001]. A few works on monitoring and fault tolerant control (FTC) of these systems have been reported. In this article, we focus on a train of vehicles. Several researches deal with longitudinal and lateral control of vehicles operating in platoon. In [Chien and Ioannou, 1992], entrainment and vehicle following controllers are proposed for autonomous intelligent vehicles in both non-tight and tight vehicle following maneuvers. A nonlinear vehicle model is used for designing the controllers. The proposed vehicle following controller is designed on the basis of constant time headway policy; whereas, the proposed entrainment controller is designed on the basis of a k-factor headway policy. The proposed vehicle following controller not only provides local individual vehicle stability but also guarantees asymptotic platoon stability without the availability of feed-forward information. Furthermore, the achieved asymptotic platoon stability is shown to be theoretically robust with respect to sensor delays. The authors in [Godbole and Lygeros, 1994] present longitudinal control laws for vehicles moving in an Intelligent Vehicle Highway System (IVHS). In particular, the scenario where cars move along the highway in tightly spaced platoons is considered. They present control schemes, based on PID control- 
ler, that perform the major longitudinal tasks that will be required from the lead vehicle of a platoon moving on an automated highway. More specifically, schemes that maintain safe spacing, track an optimal velocity and perform various maneuvers (forming, breaking up platoons and changing lanes) are described. In [Swaroop and Rajagopal, 2001], the authors analyze the problem of stability of a vehicle string in the presence of parametric uncertainty and present Lyapunov-based decentralized adaptive control algorithm to compensate such parametric variations. They examine this direct adaptive longitudinal control algorithm for platoon performance and parameter convergence. Other works concerning longitudinal control of vehicles in platoon can be found in [Sheikholeslam and Desoer, 1993], [Lee and Kim, 2002] and [Li and Bai, 2009]. Concerning lateral control of a platoon of vehicles, in [Lee et al., 1999], a lateral controller is implemented by using a fuzzy-like gain-scheduled PD control algorithm. Magnetic sensors are used to extract the lateral deviation from the magnetic field induced by an inductive wire, which indicates the center of road. Other lateral controls are proposed in [Awawdeh et al., 2005] [Malan et al., 2007][Peng et al., 2006] [Sotelo, 2003]. Meanwhile, the growing complexity of automated systems and the constraints of competitiveness in terms of production cost, availability and security, have mobilized in recent years a large community of scientists to improve monitoring and FTC systems [Blanke et al., 2006; Anwar and Chen, 2007; Biswas et al., 2003]. Monitoring mainly consists of comparing the actual behavior of the system with reference behavior describing the normal operation (for the fault detection) or describing different kinds of faults (for fault analysis and isolation), while minimizing false alarms, non-detections as well as delays in the detection of faults. The FTC system must react after detecting a fault to allow the system to continue operating in degraded mode or to stop in case of inability to continue its work [Yang et al., 2010; Dumont et al., 2006; Noura et al., 2000; Ping et al., 2008; Wang et al., 2006]. The performance degradation of these algorithms is mainly due to imperfect knowledge of model parameters values and their random variations.

This paper is organized as follows: a description and nonlinear modeling of an electric vehicle and a train of three vehicles are presented in section 2. Control of the train of vehicles operating normally is presented in section 3 . The reconfiguration of the train of vehicles in a presence of sensor or actuator faults is given in section 4 . The validity of the reconfiguration method through simulation results is provided insection5. Finally, in section6, the conclusions from this work are drawn.

\section{MODELING OF ONE VEHICLE AN D A TRAIN OF THREE VEHICLES}

Modeling is an important step for implementation of FDI and FTC algorithms. According to the objectives of monitoring, modeling must be more or less detailed. For the first time, we give the dynamic model of the used vehicle, which is an electric four-wheel steering vehicle. We present the modeling of a train of three vehicles after that.

\subsection{Description of the electric vehicle RobuCar}

The RobuCar represented in Figure 1 is an autonomous electric vehicle made by the company ROBOSOFT. The Laboratory (LAGIS) has at its disposed three prototypes that serve as a platform for experimental research. Traction of each wheel is provided by a DC motor. Braking is provided by electromagnetic brakes, which are safety brakes. It also has two steering systems, front and rear ones, based on electric jacks. Eight batteries offer autonomy of about two hours operating at maximum speed of $20 \mathrm{~km} / \mathrm{h}$.

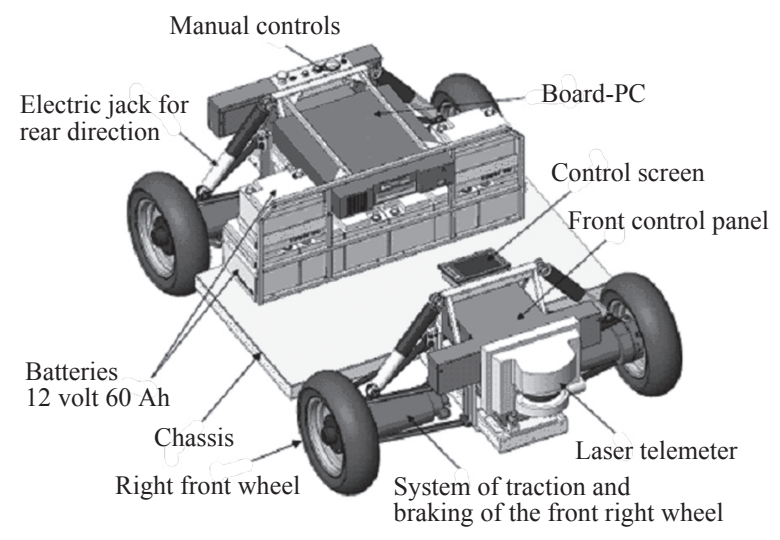

Fig. 1 RobuCar description

Sensors that are installed on the RobuCar are:

- The laser telemeter measures the distance and the direction of a potential obstacle.

- Incremental encoders (one for each wheel) measure rotational speeds of the wheels.

- Absolute encoders (one for each wheel) measure the steering angles of the wheels.

- Planar inertial sensor gives the velocities and accelerations of yaw, longitudinal and lateral motions.

- The GPS receptor gives the absolute position of the vehicle.

- Actuators are: 4 traction/braking motors (one for each wheel).

- 2 electric jacks for the steering (one for each axle)

\subsection{Modeling of the electric vehicle RobuCar}

The following assumptions are made for RobuCar modeling: 
- the pitch and roll dynamics are neglected,

- the road is supposed to be uniform so the suspension dynamics are not considered,

- the steering angles are supposed to be small $(\leq 10)$,

- the front lateral forces are equal and the rear lateral forces are equal.

The dynamics considered are: dynamics of 4 wheels, longitudinal and lateral dynamics, front and rear dynamics and yaw dynamics.

The model of the vehicle is given by the state space representation as follow:

$$
\left\{\begin{array}{c}
\dot{x}=f(x)+B u \\
y=C x
\end{array}\right.
$$

Where $f(x)$ is a known nonlinear function which represents the dynamics of the vehicle and depends on model parameters given in [Bouibed et al., 2009].

The state vector $x$ is:

$$
x=\left[\omega_{1} \omega_{2} \omega_{3} \omega_{4} v_{x} d_{x} v_{y} d_{y} \dot{\alpha}_{f} \alpha_{f} \dot{\alpha}_{r} \alpha_{r} \Gamma\right]^{T}
$$

Where $\omega_{i}$ is the angular velocity of wheel $i, v_{x}$ and $v_{y}$ are the longitudinal and lateral velocities, $d_{x}$ and $d_{y}$ the longitudinal and lateral position, $\alpha_{f}$ and $\alpha_{r}$ are the front and rear steering angles, $\dot{\alpha}_{f}$ and $\dot{\alpha}_{r}$ the front and steering velocities, and $\Gamma$ the yaw angle.

The input vector is:

$u=\left[\begin{array}{llllll}u_{1} & u_{2} & u_{3} & u_{4} & u_{f} & u_{r}\end{array}\right]^{T}$

Where $u_{i}$ is the engine torque applied in wheel $i$ and $u_{f}$ and $u_{r}$ are the front and rear engine torque of the steering angle.

The measured vector $y$ is constituted by the angular velocities, the longitudinal velocity and position, the lateral velocity and position and the yaw angle.

\subsection{Modeling of a train of vehicles}

The principle is to have a leading vehicle, for which the trajectory is imposed. Then the other vehicles follow automatically the path of the leader while maintaining a fixed space between them. Each following vehicle with the laser telemeter measures the distance that separates it from his predecessor and the angle between it and the vehicle in front. Based on these two measures, one acts on the acceleration/braking to maintain fixed the inter-vehicular distance and on the steering to track the trajectory of the leader.

We use the same model of one vehicle (1) for the dynamic modeling. The followers are presented by this model, for which we add dynamics of the intervehicular distance $e_{x i}$ and the orientation angle $\Phi_{i}$. The inter-vehicular spacing (Figure 2) measured by the laser telemeter is given by:

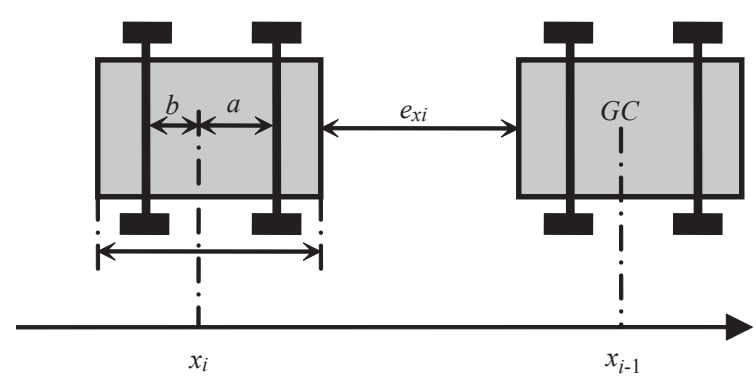

Fig.2 Inter-vehicular distances

$e_{x i}=d_{x(i-1)}-d_{x i}-L(i=1,2)$

where $d_{x i}$ and $d_{x(i-1)}$ are respectively the longitudinal position of the $i^{\text {th }}$ and the $(i-1)^{\text {th }}$ follower vehicle and $L$ is the length of each vehicle.

$\Phi_{i}=\psi_{i-1}-\psi_{i}$

where $\Psi_{i}$ is the yaw angle of $i^{\text {th }}$ follower vehicle. The $i^{\text {th }}$ follower vehicle is represented by the following model:

$\left\{\begin{array}{c}\dot{X}_{i}=f_{i}\left(X_{i}\right)+B_{i} U_{i} \\ Y_{i}=C_{i} X_{i}\end{array}\right.$

$X_{i}$ is the state vector constituted by the initial state vector $x$ and the distance $d_{x i}$ and the orientation angle $\Phi_{i}$.

\section{CONTROL OF A TRAIN OF VEHICLES IN NORMAL OPERATION}

In this section, we focus on the normal operation of the train. The strategy is based on tracking the reference trajectory and maintaining a safe distance between vehicles. We use the laser telemeter measurements of the controlled vehicle, which are the distance and the direction of the vehicle in front. This paper adopts the method of checking the behavior of the vehicle immediately in front. The leading vehicle is given a trajectory that it follows. The rest of the vehicles that are behind it follow the behavior of the leading vehicle by checking various parameters such as the inter-vehicular distance or the relative velocity between the vehicles. These values are then compared with the specified constant value. If the measured value deviates from the corresponding value, the necessary corrective measures are automatically applied to the vehicle's behavior.

\subsection{Longitudinal control}

In this paper, the inter-vehicular distance is constantly measured and compared with a constant value that is obtained taking into account all the factors that come 
into play while driving a car such as vehicular capabilities, reaction times, road surfaces etc.

Also, to prevent slickness [Swaroop et al., 2001] and other oscillatory faults, the speeds of all the vehicles are kept the same. If the inter-vehicular distance deviates from the specified constant, the following vehicle executes the necessary manoeuvres to bring the distance to the safe value. For example, if the value goes below the safety distance, then there is a chance of a crash between the vehicles if any problem occurs. Therefore, the following vehicle will decrease its speed, i.e. decelerate until the distance between the vehicles comes back to the safe value, after which it again accelerates to the speed of the leading vehicle so as to keep the system moving. Also, a deviation from the safe value of the inter-vehicular distance is also taken in case of malfunctioning of the sensors or the vehicle. This ensures more than enough space for the following vehicle to take evasive measures and prevent any mishap from happening. A second order sliding mode technique [Levant, 1993] is used for longitudinal control. This is achieved by applying the algorithm not directly on the control input $u$ but on its derivative $\dot{u}$. This method is adapted for nonlinear system and permits a considerable reduction of the inherent chattering phenomenon. Besides, sliding mode controllers are robust to sensor noise which is very important for experiments knowing that several sensors are required for longitudinal control.

The desired inter-vehicular distance between the $i^{\text {th }}$ and the $(i-1)^{\text {th }}$ vehicle, is given by the following expression:

$e_{d i}=d_{s}+\tau v_{x i}$

where $d_{s}$ represents the distance between two successive vehicles at stop and it is constant, $\tau$ is a positive coefficient fixed by the operator and corresponds to the reaction time of the driver and $v_{x i}$ is the longitudinal velocity of the vehicle $i$.

We chose the sliding surface $S_{i}$ as the difference between the desired safety distance $e_{d i}$ and the distance given by the laser telemeter $e_{x i}$. This surface is given by the following equation:

$S_{i}=e_{d i}-e_{x i}$

By replacing $e_{d i}$ by its expression given in (5), we obtain:

$S_{i}=\left(d_{s}+\tau v_{x i}\right)-e_{x i}$

The control inputs applied on the four wheels are the motor's torques, but for simplification, it is preferable to use the rotation velocities of the wheels as inputs because they are easy to measure. To control the $i^{\text {th }}$ follower vehicle depending on the leader velocity, the desired control inputs applied to the four wheels are deduced from the dynamics of the 4 wheels.

By considering that the four wheels roll have the same velocity, we obtain:

$v_{x i}=R \omega_{i}$

The Twisting algorithm [Levant, 1993] is applied on the follower vehicles to follow automatically the leader vehicle. Historically, this algorithm is the first known second order sliding mode. This algorithm was just developed to control systems with relative degree one in order to avoid chattering. The control law depends of an equivalent and can be found by the resolution of the derivative of sliding surface around the ori$\operatorname{gin} \dot{S}_{i}=0$. The gains of the control law are determined to satisfy the application conditions of the Twisting algorithm which converges in finite time. These conditions can be found in [Bouibed et al, 2008].

The equivalent control is given by :

$u_{i_{-} e q}^{*}=\frac{\dot{e}_{x i}}{\tau R}$

$R$ is radius of wheel.

\subsection{Lateral Control}

We develop a reference trajectory tracking that requires all vehicles after developing longitudinal controller to maintain safe inter-vehicular spacing. The aim of this method is to generate the reference trajectory imposed for all vehicles. The lateral position of the reference trajectory $d_{y i \text { ref }}$ is assumed to be expressed as a function of measured longitudinal position of the $i^{\text {th }}$ vehicle, where $d_{x i}$ is given by the GPS.

$d_{y i \_r e f}=f\left(d_{x i}\right)$

Thus the trajectory is calculated in real time regardless of time and velocity of the vehicle. The advantage of this method is that it allows vehicles followers to avoid cut corners that is the problem of controllers using as error the difference between the lateral position of the leader and follower.

Trajectory planning is used in [Dumont et al., 2006] in the case of one vehicle and we extend it to a train of vehicles in our case. We use a steering control with error as the difference between the desired lateral position $d_{y i \text { ref }}$ and the measured lateral position $d_{y i}$.

$e_{y i}=d_{y i}-d_{y i-r e f}$ 


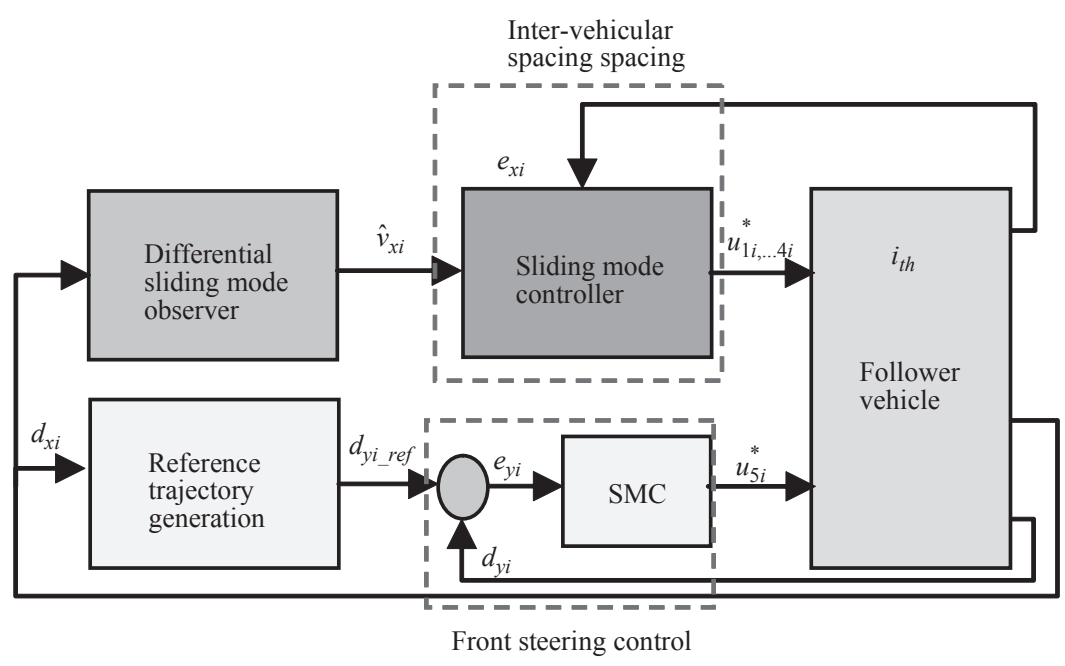

Fig. 3 Longitudinal and lateral control of follower vehicle

We use also a sliding mode control similar to that used for the longitudinal control described previously for the lateral control. So, the desired input applied to the front steering system of the $i^{\text {th }}$ follower vehicle is:

Figure 3 shows the general simulation scheme of the longitudinal and lateral control of the follower vehicle.

\section{RECONFIGURATION OF PLATOON OF VE- HICLES IN PRESENCE OF FAULTS}

This section is devoted to the reconfiguration of a train of vehicles when a fault occurs in one of the three vehicles.

Firstly, we study the various degraded modes that may appear due to the different faults in the vehicles of the convoy. Then, we will develop strategies for reconfiguration adapted for each fault.

Modeling of the train of vehicles shows equations relating to each vehicle and relationships between vehicles. The application of monitoring methods on this model would establish a central monitoring system.

This system is supplied by all the sensor information of each vehicle, so as to detect and to isolate faults. This solution is obviously not possible, monitoring can only be distributed. Each vehicle has its own monitoring system. Thus in each vehicle, we implement monitoring algorithms obtained using parity space and observer approaches. The detail of these methods can be found in [Bouibed et al., 2009a, 2009b, 2010].

These monitoring methods allow us to detect faults in a vehicle. We must reconfigure the vehicle to continue its mission depending on the nature of the fault. In some cases, reconfiguration is not possible and the fault may lead to a vehicle stop. We distinguish the following three cases:

- Case A: Fault, for which the control is tolerant and
Table 1 Different fault situations and reconfiguration strategies

\begin{tabular}{|c|c|c|l|}
\hline$V_{1}$ & $V_{2}$ & $V_{3}$ & Strategies \\
\hline $\mathrm{N}$ & $\mathrm{N}$ & $\mathrm{N}$ & Normal operation of the train \\
\hline $\mathrm{A}$ & $\mathrm{N}$ & $\mathrm{N}$ & Multi observers for sensor faults and \\
\hline $\mathrm{N}$ & $\mathrm{A}$ & $\mathrm{N}$ & control switching for \\
\hline $\mathrm{N}$ & $\mathrm{N}$ & $\mathrm{A}$ & actuator faults \\
\hline $\mathrm{B}$ & $\mathrm{N}$ & $\mathrm{N}$ & $\begin{array}{l}\text { Reconfigure the leader and adapt the } \\
\text { follower controllers }\end{array}$ \\
\hline $\mathrm{N}$ & $\mathrm{B}$ & $\mathrm{N}$ & Adapt the vehicles controllers \\
\hline $\mathrm{N}$ & $\mathrm{N}$ & $\mathrm{B}$ & Use inter-vehicular distance \\
\hline $\mathrm{C}$ & $\mathrm{N}$ & $\mathrm{N}$ & Reconstruction of a new train \\
\hline $\mathrm{N}$ & $\mathrm{C}$ & $\mathrm{N}$ & And new necessary controls \\
\hline $\mathrm{N}$ & $\mathrm{N}$ & $\mathrm{C}$ & $\begin{array}{l}\text { Abandon of } V_{3} \text { and constitute the train } \\
\text { with } V_{1} \text { and } V_{2}\end{array}$ \\
\hline
\end{tabular}

$\mathrm{N}$ : normal operation

$V_{i}$ : vehicle $i$

A: case A, B: case B and C: case C

thus for which there is no impact on the trajectory and the velocity of the vehicle. This is illustrated by some sensor and actuator faults.

- Case B: Fault that leads to an alteration of the velocity of a vehicle or a small disturbance of the trajectory. This is illustrated by two faults: a position sensor fault (GPS) and a traction motor fault.

- Case C: Fault, which does not allow the vehicle to continue the mission, the vehicle stops or locking actuator making it impossible to follow the trajectory.

\section{SIMULATION RESULTS}

In this section, we give the results obtained by simu- 
lation of various algorithms presented above using Matlab/Simulink software. At first, we simulate a train of three vehicles operating without faults with the generation of the reference trajectory for each vehicle. In this case, we use front steering control and the inter vehicular distance control. Then, we simulate a fault in the front steering system of the leader vehicle. In this case, the lateral control will automatically switch to the rear steering control.

\subsection{Reference trajectory tracking without fault}

All vehicles follow the reference trajectory using the front steering in normal operation. The safety distances between vehicles are maintained with the sliding mode controllers using the laser telemeter data.

Figure 4 shows the reference trajectory and the trajectories of three vehicles. Note that this reference trajectory is followed by three vehicles and that safety distances between vehicles are maintained at the desired distances as shown in Figure 5. Initially, the vehicles are placed one meter behind the other and after a short transient time, necessary for the stabilization of the control, the inter-vehicular distances are stabilized at around $5.2 \mathrm{~m}$.

Figures 6 and 7 show the control inputs to the front and rear steering systems of three vehicles. It is noted

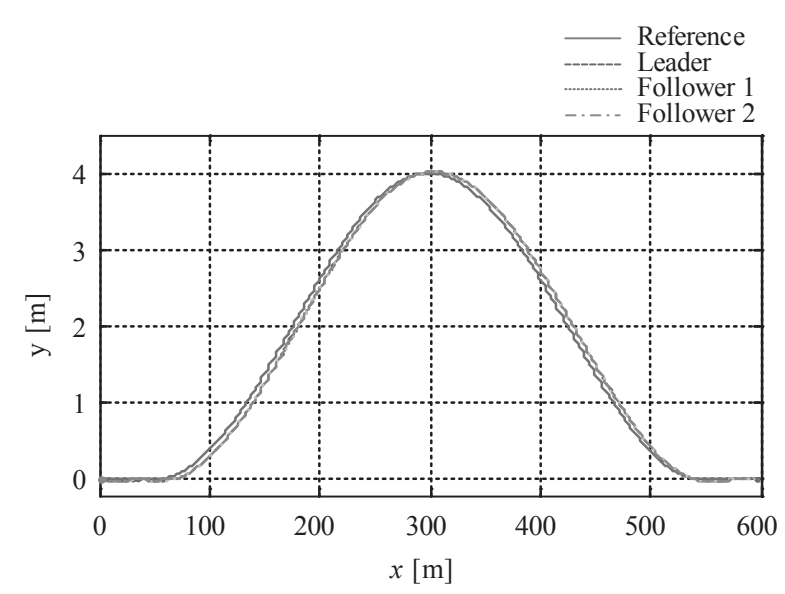

Fig. 4 Reference trajectory tracking
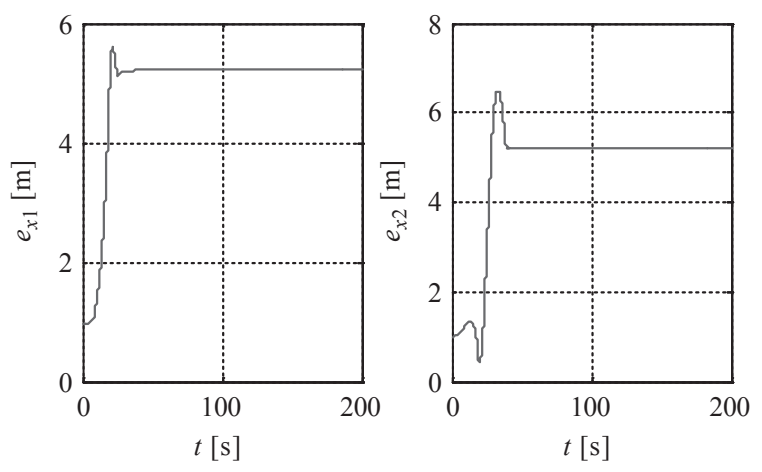

Fig. 5 Inter-vehicular distances
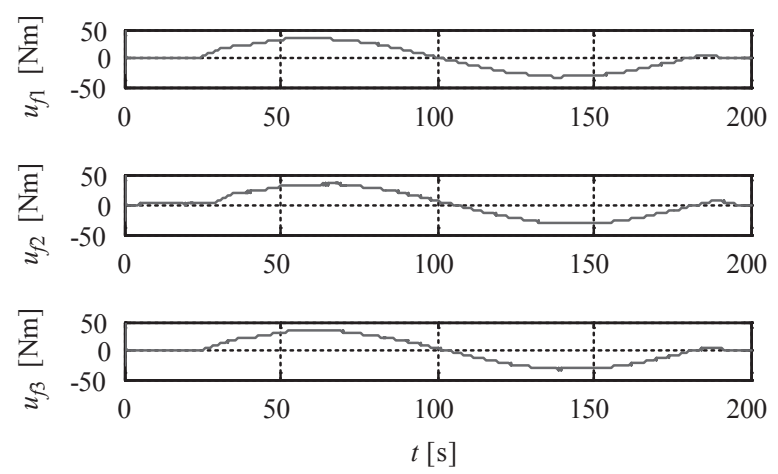

Fig. 6 Front steering controls of three vehicles
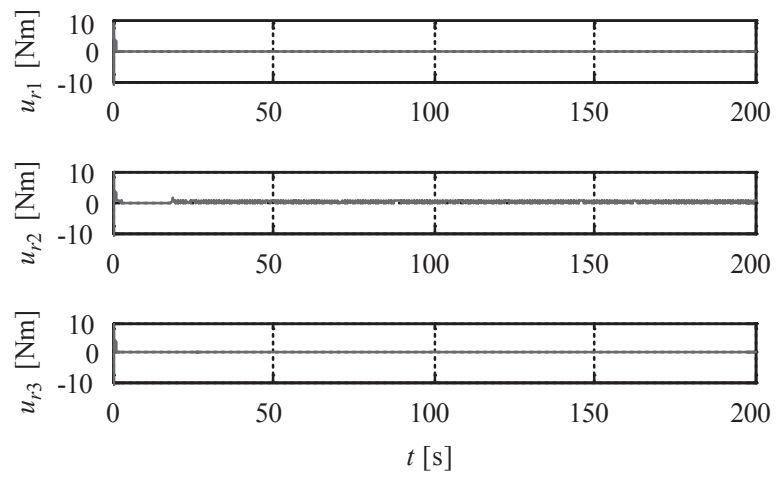

Fig. 7 Rear steering controls of three vehicles

that only front steering controls are used and those of rear steering are zero.

\subsection{Fault on front steering actuator of leader vehicle} In this part, a fault in the front steering system of the leader vehicle is simulated at the moment $t=150 \mathrm{~s}$. When this fault occurs, the lateral controller of the leader switches automatically to the rear steering control.

Figure 8 shows the trajectories of three vehicles, which follow the reference trajectory. One notes that

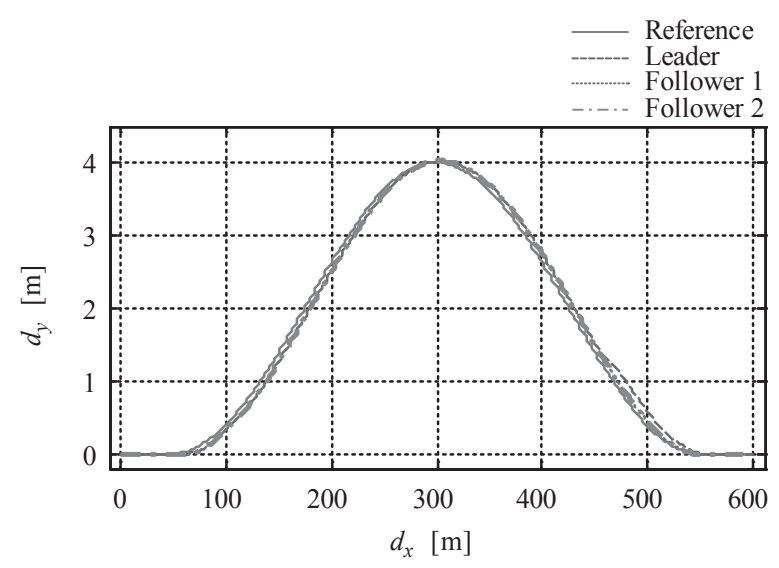

Fig. 8 Reference trajectory tracking with a fault on front steering actuator of leader 
there is a small deviation of leader vehicle trajectory from the reference at the time of fault occurrence. But, this deviation is corrected after little time by the rear steering system.

Regarding the safety distances, they are kept constant as shown in Figure 9.
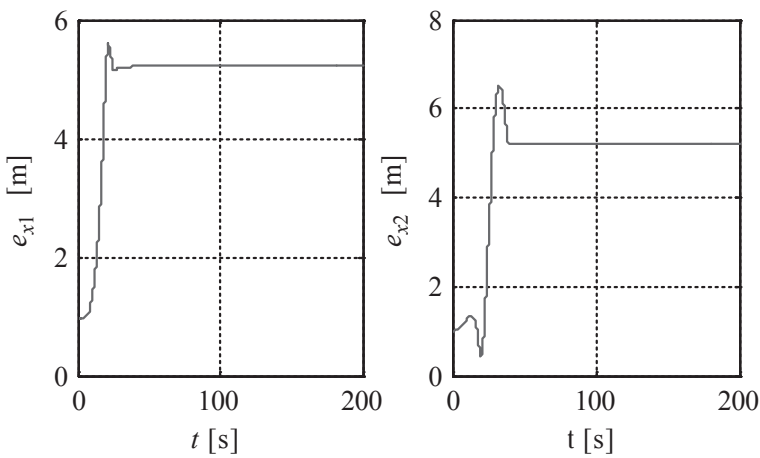

Fig. 9 Inter-vehicular distances in presence of steering actuator fault

Figures 10 and 11 show the control inputs to the front and rear steering. One notes that at the moment of occurrence of fault, the rear steering control of the leader vehicle becomes different from zero. This shows the switching of the control to the rear steering. This fault is visible in the figure of the front steering control at the time $t=150 \mathrm{~s}$.

\section{CONCLUSION}

The results obtained by simulation of case when the train of vehicle operates with a fault in the front steering system of the leader vehicle show that the reconfiguration strategy that we have adopted gives good results. The figures show that vehicles continue to follow the reference trajectory in spite of this fault.

We observe also how the steering system has switched from the front steering to the rear steering at the instant of faults.

In the first part of this work, we have developed longitudinal and lateral controls for the train of electric vehicles operating without faults. The longitudinal control consists of maintaining the safety spacing between the vehicles at the desired distances. These distances are compared with the measured ones by laser telemeters situated on the front of each vehicle. The differences are used to calculate the control inputs using sliding mode algorithm. These controls are applied on the traction motors of each wheel. The lateral control is based on the reference trajectory generation imposed for all vehicles. The difference between the reference trajectory and the trajectory given by the GPS sensor is used to calculate the front steering control input.
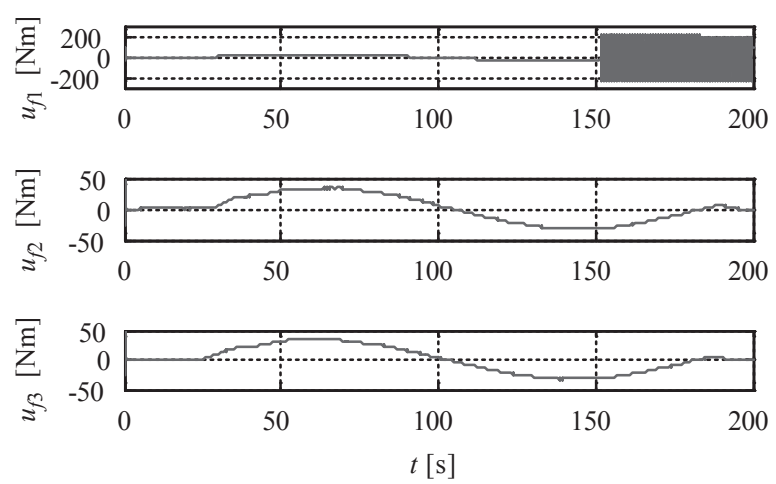

Fig. 10 Front steering controls of three vehicles in presence of steering actuator fault
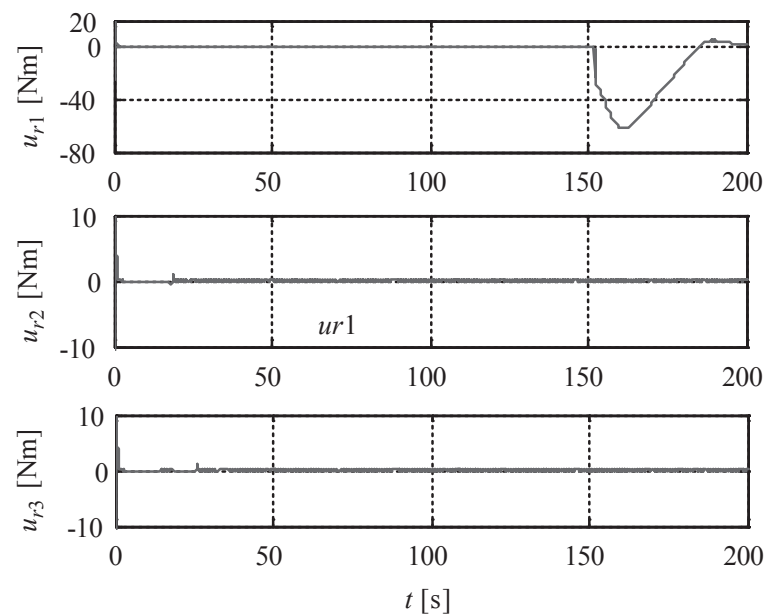

Fig. 11 Rear steering controls of three vehicles in presence of steering actuator fault

In the second part, we have proposed reconfiguration strategies for fault situations that may occur in one of the vehicles. The aim is that the combination of vehicles may continue his mission in the presence of a fault (degraded mode). These situations are classified into three categories:

- Sensor or actuator fault which has not altered the vehicle trajectory. The reconfiguration strategy is to select one observer among multiple observers for a sensor fault. The selected observer must not be affected by the sensor fault. So, controllers use no fault estimated outputs. The reconfiguration block selects the control appropriately depending on the type of actuator fault. This strategy is used for faults supposed to be known in advance.

- Fault that leads to an alteration of the velocity of a vehicle or a small disturbance of the trajectory. This is illustrated by two faults: a position sensor fault (GPS) and a traction motor fault.

- Fault, which does not allow the vehicle to continue the mission, the vehicle stops or locking actuator, makes it impossible to follow the trajectory. We 
have taken faults belonging to the first situation described above for simulations.

\section{Acknowledgements}

This work was supported by the CISIT (International Campus for Safety and Intermodality in transportation) located in the Region Nord Pas de Calais) and in the framework of INTRADE (intelligent Transportation for Dynamic Environment), Interreg IVB, 091C, North West Europe Zone.

\section{References}

Anwar, S., and L. Chen, An analytical redundancybased fault detection and isolation algorithm for a road-wheel control subsystem in a steer by wire system, IEEE Transactions on Vehicular Technology, Vol. 56, No. 5, 2859-2869, 2005.

Awawdeh, A., F. Espinosa, and M. Mazo, Non-linear trajectory generation and lateral control new algorithms to minimize platoon's oscillations, IEEE American Control Conference, Vol. 4, 3345-3350, 2005.

Barth, M., and M Todd, Intelligent transportation system architecture for a multi-station shared vehicle system, IEEE Intelligent Transportation Systems, 240-245, 2000.

Biswas, G., G. Simon, N. Mahadevan, S. Narasimhan, J. Ramirez, and G. Karsai, A robust method for hybrid diagnosis of complex systems, Proceedings of the 5th ymposium on Fault Detection, Supervision and Safety for Technical Processes, 1125-131, 2003.

Blanke, M., M. Kinnaert, J. Lunze, and M. Staroswiecki, Diagnosis and Fault Tolerant Control, Springer-Verlag, 2004.

Bouibed, K., A. Aitouche, A. Rabhi, and M. Bayart, Estimation of contact forces of a four wheel steering electric vehicle by differential sliding mode observer, AIP Conference Proceedings, Vol. 1019, No. 1, 541-546, 2008.

Bouibed, K., A. Aitouche, and M. Bayart, Nonlinear parity space applied to an electric autonomous vehicle, Journal of Energy and Power Engineering, Vol. 3, No. 12, 10-18, 2009.

Bouibed, K., A. Aitouche, and M. Bayart, Sensor and actuator fault detection and isolation using two model based approaches: Application to an autonomous electric vehicle, 18th Mediterranean Conference on Control and Automation, Marrakech, Morocco, 1290-1295, 2010.

Broggi, A., M. Bertozzi, A. Fascioli, and G. Conte, Automatic Vehicle Guidance: The Experience of the ARGO Autonomous Vehicle, World Scientific Publishing, 1999.
Chien, C. C., and P. Ioannou, Automatic vehicle following, American Control Conference, 1748-1752, 2010.

Colin, S., A. Lanoix, O. Kouchnarenko, and J. Souquières, Using cspb components: Application to a platoon of vehicles, Formal Methods for Industrial Critical Systems, Vol. 5596, 103-118, 2009.

Dumont, P., A. Aitouche, R. Merzouki, and M. Bayart, Fault tolerant control on an electric vehicle, IEEE International Conference on Industrial Technology, 2450-2455, 2006.

Godbole, D., and J. Lygeros, Longitudinal control of the lead car of a platoon, IEEE Transactions on Vehicular Technology, Vol. 43, No. 4, 1125-1135, 1994.

Lee, G., S. Kim, Y. Yim, J. Jung, S. Oh, and B. Kim, Longitudinal and lateral control system development for a platoon of vehicles, IEEE/IEEJ/JSAI International Conference on Intelligent Transportation Systems, 605-610, 1999.

Lee, G. D., and S. W. Kim, A longitudinal control system for a platoon of vehicles using a fuzzy sliding mode algorithm, Mechatronics, Vol. 12, No. 1, 97 118, 2002.

Levant, A., Sliding order and sliding accuracy in sliding mode control, International Journal of Control, Vol. 58, No. 6, 1247-1263, 1993.

Li, Z., and Q. Bai, Longitudinal distance control for vehicle following model based on tracking differentiator, IEEE International Conference on Automation and Logistics, 966- 969, 2009.

Malan, S., M. Milanese, P. Borodani, and A. Gallione, Lateral control of autonomous electric cars for relocation of public urban mobility fleet, IEEE Transactions on Control Systems Technology, Vol. 15, No. 3, 590-598, 2007.

Massot, M., J. Allouche, E. Bénéjam, and M. Parent, Praxitèle: Preliminary results from the Saint-Quentin station-car experiment, Journal of the Transportation Research Board, Vol. 1666, No. 1, 125-132, 1999.

Noura, H., D. Sauter, F. Hamelin, and D. Theilliol, Fault-tolerant control in dynamic systems: application to a winding machine, IEEE Control Systems Magazine, Vol. 20, No. 1, 33-49, 2000.

Nouvelière, L., and S. Mammar, Experimental vehicle longitudinal control using a second order sliding mode technique, Control Engineering Practice, Vol. 15, No. 8, 943-954, 2007.

Peng, Y. F., C. F. Hsu, C. M. Lin, and T. T. Lee, Robust intelligent backstepping longitudinal control of vehicle platoons with hinfinity; tracking performance, IEEE International Conference on Systems, Man and Cybernetics, Vol. 6, 4648-4653, 2006.. 
Ping, C., W. Zhengxin, and P. Ron, Novel active faulttolerant control scheme and its application to a double inverted pendulum system. Journal of Systems Engineering and Electronics, Vol. 19, No. 1, 134140, 2008.

Sheikholeslam, S., and C. Desoer, Longitudinal control of a platoon of vehicles with no communication of lead vehicle information: a system level study, IEEE Transactions on Vehicular Technology, Vol. 42, No. 4, 546-554,1993

Sotelo, M., Lateral control strategy for autonomous steering of Ackerman-like vehicles, Robotics and Autonomous Systems, Vol. 45, No. 3-4, 223-233, 2003.

Swaroop, D., J. Hedrick, and S. Choi, Direct adaptive longitudinal control of vehicle platoons, IEEE Transactions on Vehicular Technology, Vol. 50, No. 1, 150-161, 2001.

Swaroop, D., and K. Rajagopal, A review of constant time headway policy for automatic vehicle following, IEEE Intelligent Transportation Systems, 6569, 2001.

Taleb, T., A. Benslimane, and B. K. Letaief, Toward an effective risk-conscious and collaborative vehicular collision avoidance system, IEEE Transactions on Vehicular Technology, Vol. 59, No. 3, 14741486, 2010.

Wang, D., M. Pham, C. B. Low, and C. Tan, Development and implementation of a fault-tolerant vehicle-following controller for a four-wheel-steering vehicle, IEEE/RSJ International Conference on Intelligent Robots and Systems, 13-18, 2006.

Yang, H., B. Jiang, and V. Cocquempot, Fault tolerant control design for hybrid systems, Lecture Notes in Control and Information Sciences, Springer-Verlag, 2010.

(Received August 15, 2011; accepted January 10, 2012) 